DOI: https://doi.org/10.11144/Javeriana.upsy16-3. epsb

\title{
Evaluación psicológica del síndrome de burnout en profesores de educación primaria en la Comunidad de Madrid: comparación entre centros públicos y concertado*
}

\section{Psychological Assessment of Burnout Syndrome in Teachers of Primary Education in the Community of Madrid: Comparison between Public and Concerted Centers}

\author{
Eva Solera Hernández \\ Universidad Internacional de La Rioja, España \\ ORCID: http://orcid.org/0000-0002-3755-0888 \\ Sonia Gutiérrez Gómez-Calcerrada \\ Universidad Internacional de La Rioja, España \\ Domingo Palacios-Ceña \\ Universidad Rey Juan Carlos, España
}

\footnotetext{
a Autor de correspondencia. Correo electrónico: eva.solera@unir.net

Para citar este artículo: Solera Hernández, E., Gutiérrez Gómez-Calcerrada, S., \& Palacios-Ceña, D. (2017). Evaluación psicológica del síndrome de burnout en profesores de educación primaria en la Comunidad de Madrid: comparación entre centros públicos y concertados. Universitas Psychologica, 16(3), 1-9. https://doi.org/10.11144/Javeriana.upsy16-3.epsb
}

\section{RESUMEN}

El objetivo del estudio fue explorar el estado psíquico de profesores de educación primaria de varios centros públicos y concertados de la Comunidad de Madrid. Se evaluó la sintomatología ansiosa y depresiva y el síndrome de burnout, mediante la Escala HADS y el Cuestionario de Burnout del Profesorado. Los resultados indicaron que, aunque los docentes de colegios concertados reportan más sintomatología ansiosa, los profesores de colegios públicos presentan mayor presencia del síndrome de burnout en las escalas de cuestiones organizacionales, supervisión y reconocimiento. Se evidencia que los profesores de educación primaria son profesionales de alto riesgo, a pesar de que los estudios se han centrado mayoritariamente en educación secundaria.

\section{Palabras clave}

Síndrome de burnout; ansiedad; profesores de educación primaria; Comunidad de Madrid

\begin{abstract}
The study objective was to explore the psychic state of Primary Education teachers of several public and concerted centers of the community of Madrid. We evaluated anxiety and depression symptoms and burnout syndrome using the HADS scale and the Burnout Questionnaire of teachers. The results indicated that, although concerted schools teachers report more symptoms anxious, teachers of public schools have a greater presence of the Burnout syndrome in the scales of organizational issues, supervision and recognition. It is evident that the primary education teachers are high-risk professionals, despite the fact that the studies have focused mainly in Secondary Education.
\end{abstract}

Keywords

Burnout; anxiety; primary teachers; Madrid Community 
Eva Solera Hernández, Sonia Gutiérrez Gómez-Calcerrada, Domingo Palacios-Ceña.

\section{Introducción}

Desde hace tiempo se viene relacionando la actividad docente con el síndrome de burnout, considerando incluso que esta profesión puede superar a otras denominadas de riesgo, como mineros, policías, médicos, etc. (Acosta \& Burguillos, 2014; Arias \& González, 2009; Díaz, López, \& Varela, 2012; Ilaja \& Reyes, 2016). El motivo, como indican Esteras, Chorot y Sandín (2014), radica en que se trata de un trabajo vocacional, más personal y emocional. Por tanto, las diversas situaciones escolares a las que tiene que enfrentarse el maestro y las dificultades a la hora de resolverlas dan lugar a este fenómeno, por no disponer de estrategias o habilidades adecuadas (Arias \& González, 2009; Calvete, 2010; Camacho \& Arias, 2009; Díaz et al., 2012; Gantiva, Jaimes, \& Villa, 2010; Guerrero, Gómez, Moreno, García-Baamonde, \& Blázquez, 2011).

El síndrome de burnout, tal y como indican Moriana y Herruzo (2004, p. 598), consiste en "una respuesta de estrés crónico formada por tres factores fundamentales: cansancio emocional, despersonalización y baja realización personal". Dicha problemática es prevalente en diversas profesiones, entre ellas la docente. Así, el problema ha suscitado el interés de numerosos investigadores en el territorio español, centrándose básicamente en profesores de educación secundaria (Beltrán, Pando, \& Pérez, 2004; Gantiva et al., 2010). Otros trabajos (Arias \& González, 2009; Camacho \& Arias, 2009) plantean que esta problemática es común a todo el personal docente, independientemente del curso académico.

Entre las causas principales destacan la indisciplina (Calvete, 2010; Camacho \& Arias, 2009; Gómez-Restrepo, Rodríguez, Padilla, \& Avella-García, 2009; Pizarro, 2010; Yuste, 2007), la poca motivación del alumnado (Calvete, 2010), los conflictos entre alumnos (Extremera, Rey, \& Pena, 2010; Sánchez, 2010), la escasa implicación de las familias y la sociedad en el proceso educativo (Instituto de Evaluación y Asesoramiento Educativo [IDEA] \& Ararteko, 2006; Mestres, 2012; Pizarro, 2010; Sánchez,
2010) y otros factores relacionados con aspectos laborales y organizativos del centro (Latorre \& Sáez, 2009; Pizarro, 2010; Yuste, 2007).

Como podemos ver, los problemas de convivencia escolar y la situación familiar y social actual tienen una repercusión clara en el estado de salud psíquica de los docentes, al lidiar con diferentes conflictos y a veces no disponer de estrategias o habilidades para resolverlos. Además, se ha producido un cambio a nivel social y familiar en el cuidado y la educación de los hijos, delegándose estas funciones en el centro educativo. Por tanto, los profesores se ven sobrecargados y tienen que asumir funciones que anteriormente no realizaban, lo cual afecta su salud física y psíquica (Arias \& González, 2009; Camacho \& Arias, 2009; Extremera et al., 2010; Giron (s. f.); Guerrero et al., 2011; Pizarro, 2010).

En este contexto, tienen mucha importancia los aspectos organizacionales (Briones, Tabernero, \& Arenas, 2010), que incluyen

clima laboral, satisfacción y sobrecarga laboral, trabajos administrativos, clases con muchos alumnos, conflicto de rol, problemas con los superiores, compañeros y padres de alumnos, legislaciones educativas, desarrollo profesional deficitario, salarios bajos, trastornos de conducta y conductas disruptivas de los alumnos (Briones, Tabernero, \& Arenas, 2010 como se citó en Díaz et al., 2012, p.219). En concreto, Díaz et al. (2012) destacan la importancia de las condiciones organizacionales y la supervisión, principalmente el apoyo recibido; así como el estrés del rol, el exceso de trabajo administrativo, los recursos y materiales disponibles para la labor docente.

Además, como indican Ibáñez et al., (2012), en Colombia, con la reforma educativa introducida por la Ley 715 de 2001, cambia el sistema de distribución de los docentes en los centros y se determinan los recursos asignados a los centros públicos, lo que ha provocado mayor sobrecarga para los docentes, aumento del número de alumnos en las aulas, así como espacios y recursos reducidos (Diazgranados, González \& Jaramillo, 2006, como se citó en Ibáñez et al., 2012). 
Del mismo modo, dada la actual situación educativa española, inmersa en un nuevo proceso de reforma, con importantes repercusiones a nivel laboral, consideramos de gran relevancia analizar el estado de salud de los docentes y sus repercusiones en las aulas. No debemos olvidar la importante relación entre síndrome de burnout y ansiedad y depresión, que incrementa este tipo de trastornos.

Por todo lo mencionado anteriormente, como recogen Ibáñez et al. (2012), se han realizado diferentes estudios en Colombia (Aguilar, Barros $\&$ Caro, 2011; Diazgranados et al., 2006; Mena, 2011; Padilla et al., 2009; Guevara y SánchezLozano, 2014 como se citó en Ibáñez et al., 2012), México (Camacho y Arias, 2009; León, Matsui, \& Beltrán, 2008 como se citó en Ibáñez et al., 2012) y Argentina (Bergadá et al., 2005 como se citó en Ibáñez et al., 2012), para estudiar el síndrome de burnout y tomar medidas al respecto. Del mismo modo, Ratto, García Pérez, Silva y González (2015) han estudiado la situación en Uruguay; Arias y Jiménez (2013) en Perú; Jiménez, Jara y Miranda (2012) en Chile, y en Colombia; por citar algunas de las muchas investigaciones realizadas sobre este tema.

Este estudio se planteó con el objetivo de evaluar la sintomatología relativa al síndrome de burnout, ansiedad y depresión en el profesorado de educación primaria, por ser un colectivo que no ha sido analizado exhaustivamente, puesto que mayoritariamente las investigaciones se han centrado en las etapas educativas posteriores. Además, se pretendía comparar la situación de los docentes en función del tipo de centro donde desempeñaban su labor. En concreto, centros públicos y centros concertados ${ }^{1}$.

\section{Método y materiales}

\section{Participantes}

Para llevar a cabo la investigación, contamos con una muestra incidental de 48 profesores (30 de colegios concertados y 18 de colegios públicos) de 8 centros de Madrid capital y 2 de otras poblaciones de la Comunidad. La media de edad fue 40.6 años (43.69 en concertados y 35.66 en públicos).

Los criterios de inclusión fueron: 1) impartir docencia en quinto y sexto curso de educación primaria, por ser cursos cercanos a la etapa de educación secundaria; 2) tener como mínimo un año de experiencia como docentes en dichos cursos, y 3) estar en activo en el momento del estudio. Dichos criterios fueron comunicados en el primer contacto con los diversos centros, para iniciar el reclutamiento de los participantes.

En cuanto a su experiencia docente, la media total fue de 16.12 años, 19.63 en centros concertados y 12.27 en centros públicos. Respecto a su situación laboral, el 31.25 \% eran funcionarios, el $41.66 \%$ poseía un contrato indefinido, el $6.25 \%$ corresponde a interinos y el $4.16 \%$ a personal de relevo.

\section{Instrumentos}

Se utilizaron los siguientes instrumentos de evaluación:

Escala de Ansiedad y Depresión en Hospital (The Hospital Anxiety and Depression Scale [HADS]; Zigmon \& Snaith, 1983), en su versión española, para detectar estados de depresión y ansiedad. La versión española de 14 ítems, 7 para la subescala de ansiedad y 7 para la de depresión, fue desarrollada por Tejero, Guimerá, Farré, y Peri (1986), con una consistencia interna de 0.81 para la subescala de ansiedad y 0.82 para la de depresión.

Cuestionario de Burnout del Profesorado (CBP-R; Moreno-Jiménez, Garrosa, \& González, 2000), para detectar presencia del síndrome de burnout en los docentes. Es una versión revisada del Cuestionario de Burnout del Profesorado (CBP; Moreno-Jiménez, Oliver, \& Aragoneses, 1993). Consta de 3 factores, con sus correspondientes escalas: Desorganización (escalas de Supervisión y Condiciones Organizacionales) y Problemática Administrativa (escalas de Preocupaciones Profesionales y Falta de Reconocimiento Profesional), como factores antecedentes, y Estrés y Burnout (escalas de Agotamiento 
Emocional, Despersonalización y Falta de Realización), como consecuentes. En total 66 ítems y varias preguntas sobre datos sociodemográficos. La consistencia interna presenta valores entre 0.61 y 0.87 , según la escala.

\section{Procedimiento}

Se utilizó un diseño no experimental mediante un estudio de tipo comparativo descriptivo. Se estableció contacto con diversos centros educativos de educación primaria de la Comunidad de Madrid para explicarles el objeto del estudio y pedirles su colaboración voluntaria. Se realizó una reunión informativa con los centros que quisieron participar, tanto con la dirección como con los profesores interesados en la participación del estudio. En dicha reunión, se informó sobre las instrucciones de cumplimentación de los instrumentos seleccionados. Además, se les ofreció la posibilidad de una segunda reunión para la cumplimentación de los instrumentos con los autores del estudio, o bien, complementarlos en su tiempo libre con el objetivo de no interferir en el desempeño laboral.

\section{Resultados}

En primer lugar, se realizó un análisis descriptivo de la muestra reclutada. En la Tabla 1 se recogen las medias y desviaciones típicas (S. D.) de las variables estudiadas (sociodemográficas, ansiedad, depresión y síndrome de burnout) en los dos tipos de centros (públicos y concertados) que participaron en el estudio.
TABLA 1

Medias y desviaciones típicas de las variables estudiadas

\begin{tabular}{lcccc}
\hline \multirow{2}{*}{\multicolumn{1}{c}{ Variables }} & \multicolumn{4}{c}{ Centros Públicos $(\mathrm{N}=18)$ entros Concertados $(\mathrm{N}=3$} \\
\cline { 2 - 5 } & Media & S. D. & Media & S. D. \\
\hline Edad & 35.7 & 7.8 & 43.7 & 12.5 \\
Experiencia & 10.3 & 7.3 & 19.6 & 11.9 \\
Hijos & 0.8 & 0.9 & 0.7 & 0.9 \\
Personas a cargo & 0.6 & 0.9 & 0.2 & 0.5 \\
\hline HADS & & & & \\
\hline Ansiedad & 3.3 & 2.1 & 5.2 & 2.7 \\
Depresión & 2.3 & 2.2 & 2.3 & 2.6 \\
\hline Burnout & & & & \\
\hline Estrés & 2.5 & 0.7 & 2.4 & 0.7 \\
Agotamiento & 1.9 & 0.6 & 1.9 & 0.6 \\
Despersonalización & 1.5 & 0.5 & 1.4 & 0.5 \\
Realización & 1.9 & 0.7 & 1.7 & 0.5 \\
Supervisión & 2.8 & 0.5 & 2.4 & 0.7 \\
Competencias Organizacionales & 2.7 & 0.4 & 2.4 & 0.5 \\
Preocupaciones Profesionales & 2 & 0.7 & 1.6 & 0.6 \\
Reconocimiento & 2.9 & 0.7 & 2.2 & 0.9 \\
\hline
\end{tabular}

Fuente: elaboración propia.

Para analizar las diferencias entre los dos tipos de centros en las variables evaluadas con los instrumentos, se utilizó una prueba t de student de muestras independientes. Previamente se realizó una prueba $F$ (ANOVA de muestras independientes) para analizar la varianza de las dos muestras.

En la Tabla 2 se muestran los resultados del análisis de varianza para todas las variables estudiadas en las dos muestras. Como se puede apreciar, no existen diferencias estadísticamente significativas en las varianzas.

TABLA 2

ANOVA de muestras independientes

\begin{tabular}{|c|c|c|}
\hline Variables & $\mathrm{F}$ & Significación* \\
\hline \multicolumn{3}{|l|}{ HADS } \\
\hline Ansiedad & 0.55 & No significativo \\
\hline Depresión & 0.69 & No significativo \\
\hline \multicolumn{3}{|l|}{ Burnout } \\
\hline Estrés & 0.92 & No significativo \\
\hline Agotamiento & 1.09 & No significativo \\
\hline Despersonalización & 1.09 & No significativo \\
\hline Realización & 1.90 & No significativo \\
\hline Supervisión & 0.49 & No significativo \\
\hline Competencias Organizacionales & 0.75 & No significativo \\
\hline Preocupaciones Profesionales & 1.41 & No significativo \\
\hline Reconocimiento & 0.66 & No significativo \\
\hline
\end{tabular}

En la Tabla 3 se muestran los resultados de la prueba $t$ en cada una de variables analizadas y el tamaño de efecto de las diferencias analizadas. 
TABLA 3

Prueba $t$ de student de muestras independientes y tamaño del efecto

\begin{tabular}{|c|c|c|c|}
\hline Variables & $t$ & Significación* & T. E. \\
\hline \multicolumn{4}{|l|}{ HADS } \\
\hline Ansiedad & 2.43 & $\mathrm{p}<0.02$ & 0.75 \\
\hline Depresión & 0.45 & No significativo & 0 \\
\hline \multicolumn{4}{|l|}{ Burnout } \\
\hline Estrés & 0.71 & No significativo & 0.14 \\
\hline Agotamiento & 0.15 & No significativo & 0 \\
\hline Despersonalización & 0.55 & No significativo & 0.2 \\
\hline Realización & 1.10 & No significativo & 0.32 \\
\hline Supervisión & 2.08 & $\mathrm{p}<0.05$ & 0.65 \\
\hline Competencias Organizacionales & 2.11 & $\mathrm{p}<0.05$ & 0.7 \\
\hline Preocupaciones Profesionales & 1.88 & No significativo & 0.6 \\
\hline Reconocimiento & 2.85 & $\mathrm{p}<0.01$ & 0.86 \\
\hline
\end{tabular}

* Nivel de significación: $\mathrm{p}<0.05$.

T. E.: tamaño del efecto de

las diferencias encontradas

Fuente: elaboración propia.

En cuanto a la subescala de ansiedad del HADS, podemos apreciar en la Figura 1 que los profesores de los centros concertados presentan mayor ansiedad. Esta diferencia es estadísticamente significativa con respecto a los centros públicos, tal y como se apreciaba en la Tabla 3.

Figura 1.

Puntuaciones medias de las variables estudiadas por tipo de centro

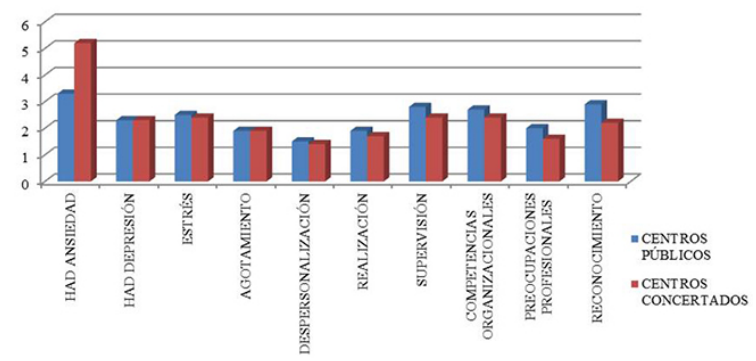

Fuente: elaboración propia.

En el resultado global, considerando el total de los centros, observamos que la media en la subescala de ansiedad del HADS es de 4.47, habiendo 5 profesores de centros concertados que obtuvieron puntuaciones entre 8 y 10 , siendo 8 el punto de corte para establecer morbilidad ansiosa (véase Tabla 2). Sin embargo, en la subescala de depresión del HADS no se aprecian diferencias estadísticamente significativas entre ambos tipos de centros. En este caso, la media global de la subescala de depresión del HADS, considerando ambos tipos de centro, es de 2.31. Pero, de nuevo, encontramos profesores de centros concertados (3) que obtuvieron puntuaciones entre 8 y 9 , siendo 8 el punto de corte para presencia de sintomatología moderada o presencia de posible trastorno.

En relación con los factores analizados por el CBP-R, observamos que existen diferencias estadísticamente significativas en las escalas de Condiciones Organizacionales, Supervisión y Falta de Reconocimiento, como muestra la Figura 1. Obteniendo mejores puntuaciones los centros concertados en las tres.

Concretamente, los centros públicos presentan peor puntuación en Competencias Organizacionales (2.69), Supervisión (2.79) y Falta de Reconocimiento (4.16) que los concertados (2.42, 2.41 y 2.20 , respectivamente).

En el resto de factores analizados por el CBP$\mathrm{R}$ no se aprecian diferencias estadísticamente significativas. Sin embargo, la puntuación en todas las escalas, salvo en Despersonalización, muestra que existen participantes que han obtenido puntuaciones elevadas, presentando los centros públicos peores resultados en todas las escalas del instrumento.

Por otro lado, en la Tabla 3 podemos observar que en aquellas variables que se han encontrado diferencias estadísticamente significativas se obtiene un tamaño del efecto de medio a alto. Así, las diferencias encontradas en la variable Reconocimiento alcanzan un tamaño del efecto elevado, y las diferencias obtenidas en las variables Ansiedad, Supervisión, Competencias Organizacionales presentan un tamaño del efecto medio.

\section{Discusión}

Los resultados obtenidos indican que realmente, como han comentado diferentes investigadores (Acosta \& Burguillos, 2014; Arias \& González, 2009; Díaz et al., 2012; Ilaja \& Reyes, 2016), la población docente es un colectivo con alto riesgo de presentar problemas patológicos de ansiedad y estrés. En este aspecto, parece ser 
el profesorado de los centros concertados el que presenta mayor nivel de ansiedad (con una puntuación media de 5.16 y 5 participantes con puntuaciones moderadas).

Respecto al nivel de síndrome de burnout, también se observan diferencias entre ambos tipos de centro en las escalas de Condiciones Organizacionales, Supervisión y Falta de Reconocimiento, presentando mayores puntuaciones, y por tanto peores resultados, los centros públicos que los concertados. Estos datos parecen estar directamente relacionados con la situación laboral y económica en que se encuentra el personal docente, tal y como indican Moreno-Jiménez et al. (2000), puesto que es más frecuente que los funcionarios se quejen de un menor reconocimiento. Quizá disponer de un puesto estable les ofrece la seguridad necesaria para exponer sus quejas y su malestar profesional. Sin embargo, Arias y Jiménez (2013) observan que son los docentes de centros privados, frente a los de gestión nacional, los que manifiestan más quejas a nivel económico, puesto que el sueldo de estos en Colombia es más bajo. Además, hay que tener en cuenta la influencia de la organización, la supervisión y el apoyo en su trabajo por parte de compañeros y superiores, y la imagen social actual de la figura del profesor (Díaz et al., 2012; Esteras, Chorot, \& Sandín, 2014; Gómez-Restrepo et al., 2009; IDEA \& Ararteko, 2006; Moreno-Jiménez et al., 2000; Pizarro, 2010; Yuste, 2007). Respecto a la supervisión inadecuada, Díaz et al. (2012), Esteras et al. (2014), Gómez-Restrepo et al. (2009) y Moreno-Jiménez et al. (2000) identificaron varios elementos que influyen en el desarrollo de burnout: entorno hostil, escaso apoyo social y preocupación por parte de los superiores y órdenes poco precisas. A esto se podrían añadir las expectativas desproporcionadas sobre la labor docente, la escasa valoración profesional, los comportamientos ambivalentes, las muestras de preferencia hacia determinadas personas, la escasa inclusión en la toma de decisiones, etc. Por otro lado, el apoyo social por parte del entorno familiar y profesional es sumamente importante, reduciendo el riesgo de burnout (Calvete, 2010). En este sentido, Latorre y Sáez (2009) indican que la percepción del personal docente sobre su situación es más negativa que la de los profesores de centros concertados porque disponen de menos apoyo social, tanto por parte del equipo docente, como de los alumnos y los familiares. Todos estos aspectos repercuten directamente en la persona, generando en muchos casos intranquilidad o inestabilidad, que se traduce en problemas de ansiedad y estrés. En este sentido, Calvete (2010), Camacho y Arias (2009) y Díaz et al. (2012), entre otros, indican que existen diversos aspectos que afectan al desarrollo de la actividad educativa, provocando alteraciones en la percepción del rol docente y generando estrés y síndrome de burnout. Aspectos como: equipamiento inadecuado, grupos grandes de alumnos en el aula, interrupciones y problemas acústicos y de organización horaria, dificultades en la relación con compañeros, director, familias y comunidad escolar. Principalmente, las investigaciones destacan la falta de apoyo de superiores e inspectores educativos como causa del síndrome de burnout, fundamentalmente en la toma de decisiones ante el incumplimiento de normas por parte de los alumnos (Esteras et al., 2014; Yuste, 2007). Esto a su vez está relacionado con una inadecuada supervisión, puesto que no recibir retroalimentación sobre los resultados de la actividad laboral puede incrementar el nivel de cansancio emocional (Calvete, 2010; Díaz et al., 2012; Esteras et al., 2014; Gómez-Restrepo et al., 2009), aunque hay estudios que no observan que este dato sea significativo (Moriana, 2002). Además, Diazgranados, González y Jaramillo (2006, como se citó en Ibáñez et al., 2012) y Moriana (2002) señalan que las sucesivas reformas educativas son un factor relevante para explicar el nivel de estrés de los docentes, al igual que la escasa valoración de sus propuestas y mejoras a nivel formativo y profesional (Moriarty, Edmonds, Martin, \& Blatchford, 2001). Por tanto, no sería extraño observar síntomas de ansiedad y estrés en esta población ante los cambios constantes en materia de reforma educativa.

Siguiendo a Ibáñez et al. (2012), MorenoJiménez et al. (2000) y Yuste (2007), podemos suponer que los buenos resultados en 
Agotamiento Emocional están relacionados con los años de experiencia, puesto que los primeros han observado que son los profesores de entre 45 y 50 años los que presentan mayor agotamiento emocional y, en nuestra muestra, la media de edad global y la media de cada tipo de centro es menor (35.7 y 43.7).

En el resto de escalas, como hemos visto, no se aprecian diferencias estadísticamente significativas en centros de uno y otro tipo, aunque los públicos presentan puntuaciones más altas en todas las escalas del instrumento de burnout. Quizá el hecho de tener un puesto de trabajo fijo hace que estos profesores se sientan más seguros y libres para quejarse y mostrar su malestar, como ya hemos comentado anteriormente. Esto puede haberse acrecentado con los recientes cambios educativos en la escuela pública y con los resultados alcanzados por el alumnado en las periódicas evaluaciones PISA. Además, no debemos olvidar, como señalaron el Instituto de Evaluación y Asesoramiento Educativo (IDEA) y Ararteko (2006), que los docentes de centros públicos de entornos de menor nivel socioeconómico destacaban estar más expuestos a faltas de respeto, conductas disruptivas y vandalismo. Sin embargo, es curioso observar que los docentes de centros concertados presentan mayor nivel de ansiedad, pero inferiores puntuaciones de síndrome de burnout que los de centros públicos, algo un tanto contradictorio. Estos resultados pueden deberse a que experimentan cierto malestar, aunque aún no lo identifican como burnout o no están en disposición de quejarse debido a su vinculación laboral con el centro. Además, como indican Latorre y Sáez (2009), las condiciones laborales en ambos tipos de centros son similares, de ahí que no haya grandes diferencias entre ellos. De todos modos, estos autores señalan que en los concertados parece haber mayor apoyo social, lo que puede compensar el mayor nivel de ansiedad detectado en nuestro estudio.

Sin duda es conveniente realizar un análisis más exhaustivo respecto a estos temas, con diversos instrumentos que permitan mayor profundidad en el estudio, tomando varias medidas a lo largo del curso escolar, y con una muestra más amplia, compuesta por docentes de centros de distinta modalidad (públicos, concertados y privados). En cualquier caso, podemos concluir que existe evidencia de que el profesorado de educación primaria presenta cierto nivel de ansiedad y síndrome de burnout, lo que los convierte en profesionales de alto riesgo. Además, dado que uno de los factores más destacados es la Falta de Reconocimiento, conviene reflexionar sobre el comportamiento de directores, profesores y familiares, así como sobre la imagen social que tenemos del maestro. Del mismo modo, como señalan Gantiva et al. (2010) y Veas (2013), es necesario contar con una estructura organizacional clara para saber cómo desempeñar la función encomendada, teniendo también la posibilidad de participar en los procesos de toma de decisiones. Así, el docente se sentirá respaldado por sus superiores, sabrá cómo actuar ante determinadas situaciones y será partícipe del funcionamiento del centro. En este sentido, Muñoz y Correa (2012) plantean proporcionar refuerzos sociales a los docentes por su trabajo, reconociéndoles públicamente su labor e incrementando el compromiso del centro hacia ellos; aparte de intentar reducir "actividades repetitivas, sobrecargas en la tarea y malas comunicaciones con las directivas" ( $p$. 238). También es fundamental asegurar la estabilidad laboral para reducir su inseguridad, puesto que, como señala el Ministerio de Educación, Cultura y Deporte de España (2010), contar con un equipo estable repercute en los resultados académicos de los alumnos. Igualmente, es necesario ofrecer al personal docente recursos adecuados para enfrentarse a las diversas fuentes de ansiedad, depresión y síndrome de burnout que le rodean; por ejemplo, fomentando la autoeficacia y la autorregulación, como señalan Merino Tejedor y Lucas Mangas (2016).

Con estas propuestas, consideramos que pueden mejorar las condiciones psicológicas de los profesionales educativos, como hemos visto, afectadas por el síndrome de burnout. 


\section{Referencias}

Acosta, M., \& Burguillos, A. I. (2014). Estrés y burnout en profesores de primaria y secundaria de Huelva: las estrategias de afrontamiento como factor de protección. International Journal of Developmental and Educational Psychology. INFAD Revista de Psicología, 1(4), 303-310.

Arias, F., \& González, M. E. (2009). Estrés, agotamiento profesional (burnout) y salud en profesores de acuerdo a su tipo de contrato. Ciencia y Trabajo, 33, 172-176.

Arias, W. L., \& Jiménez, N. A. (2013). Síndrome de burnout en docentes de Educación Básica Regular de Arequipa. Educación, 22(42), 53-76.

Beltrán, C., Pando, M., \& Pérez, M. (2004). Apoyo social y síndrome de quemarse en el trabajo o burnout: una revisión. Psicología de la Salud, 14, 78-87.

Briones, E., Tabernero, C., \& Arenas, A. (2010). Job satisfaction of secondary school teachers: Effects of demographic and psycho-social factors. Revista de Psicología del Trabajo y las Organizaciones, 26, 115-122.

Calvete, E. (2010). El estrés en profesores. Crítica, 968, 71-74.

Camacho, C., \& Arias, F. (2009). Análisis de la salud y burnout en profesores mexicanos. Ciencia y Trabajo, 33, 168-171.

Díaz, F., López, A., \& Varela, M. T. (2012). Factores asociados al síndrome de burnout en docentes de colegios de la ciudad de Cali, Colombia. Universitas Psychologica, 11(1), 217-227.

Esteras, J., Chorot, P., \& Sandín, B. (2014). Predicción del burnout en los docentes: papel de los factores organizacionales, personales y sociodemográficos. Revista de Psicopatología y Psicología Clínica, 19(2), 79-92.

Extremera, N., Rey, L., \& Pena, M. (2010). La docencia perjudica seriamente la salud. Análisis de los síntomas asociados al estrés docente. Boletín de Psicología, 100, 43-54.

Gantiva, C. A., Jaimes, S., \& Villa, M. C. (2010). Síndrome de burnout y estrategias de afrontamiento en docentes de primaria y bachillerato. Psicología desde el Caribe, 26, 36-50.

Giron (s. f.). Uno de cada cuatro profesores declara sufrir agresiones físicas o verbales en la escuela. Recuperado de http://comunidad-escolar.c nice.mec.es/787/info4.html

Gómez-Restrepo, C., Rodríguez, V., Padilla, A. C., \& Avella-García, C. B. (2009). El docente, su entorno y el síndrome de agotamiento profesional (SAP) en colegios públicos en Bogotá (Colombia). Revista Colombiana de Psiquiatría, 38(2), 279-293.

Guerrero, E., Gómez, R., Moreno, J. M., GarcíaBaamonde, M. E., \& Blázquez, M. (2011). El síndrome del quemado, modos de afrontamiento del estrés y salud mental en profesores no universitarios. Behavioral Psychology Psicología Conductual, 19(3), 557-576.

Guevara, A. C., \& Sánchez-Lozano, G. M. (2014). Estrés laboral y salud mental en docentes de primaria y secundaria. Revista Colombiana de Salud Ocupacional, 4(4), 30-32.

Ibáñez, J., López, J., Márquez, A., Sánchez, N., Flórez-Alarcón, L., \& Vera, A. (2012). Variables sociodemográficas relacionadas al Síndrome de Burnout en docentes de colegios distritales. Psychologia: Avances de la Disciplina, 6(2), 103-113.

Ilaja, B., \& Reyes, C. (2016). Burnout y estrategias de inteligencia emocional en profesores universitarios: implicaciones en la salud laboral educativa. Psicología desde el Caribe, 33(1), 31-46. Recuperado de: http://www.scielo.org.co/scielo.php?scri pt $=$ sci_arttext\&pid $=$ S0123-417X201600 0100004

Instituto de Evaluación y Asesoramiento Educativo, \& Ararteko (2006). Convivencia y conflictos en los centros educativos. Recuperado de http://213.0.8.18/portal/Educantabria/R ECURSOS/Materiales/Biblestinv/EuzKadi _Convivencia_conflicto_CenEscolares_06 .pdf 
Jiménez, A., Jara, M. J., \& Miranda, E. R. (2012). Burnout, apoyo social y satisfacción laboral en docentes. Revista Semestral da Associação Brasileira de Psicologia Escolar e Educacional, 16(1), 125-134.

Latorre, I., \& Sáez, J. (2009). Análisis del burnout en profesores no universitarios de la región de Murcia (España) en función del tipo de centro docente: público versus concertado. Anales de Psicología, 25 (1), 86-92.

Merino Tejedor, E., \& Lucas Mangas, S. (2016). La autoeficacia y la autorregulación como variables moderadoras del estrés laboral en docentes de educación. Universitas Psychologica, 15(1), 205-218. http:// dx.doi.org/10.11144/ Javeriana.upsy15-1.aavm

Mestres, L. (2012). Prevenir el estrés en el sector educativo. Recuperado de http://www.educaweb.com/noticia/2012 /07/09/prevenir-estres-sector-educativo-15 638.html

Ministerio de Educación, Cultura y Deporte de España. (2010). Educación Primaria 2007. Evaluación general del sistema educativo. Madrid: Secretaría General Técnica. Subdirección General de Documentación y Publicaciones.

Moreno-Jiménez, B., Garrosa, E., \& González, J. L. (2000). La evaluación del estrés y el burnout del profesorado: CBP-R. Revista de Psicología del Trabajo y las Organizaciones, 16(1), 331-349.

Moreno-Jiménez, B., Oliver, C., \& Aragoneses, A. (1993). Configuración específica del estrés laboral asistencial en el profesorado de Educación Media. Centro Nacional de Investigación Educativa (CIDE). Manuscrito no publicado.

Moriana, J. A. (2002). Estudio epidemiológico de la salud mental del profesorado (Tesis doctoral). Universidad de Córdoba, España.

Moriana, J. A., \& Herruzo, J. (2004). Estrés y burnout en profesores. International Journal of Clinical and Health Psychology, 4(3), 597-621.

Moriarty, V., Edmonds, S., Martin, C., \& Blatchford, P. (2001). Teaching young children: Perceived satisfaction and stress. Educational-Research, 43, 33-46.

Muñoz, C. F., \& Correa, C. M. (2012). Burnout docente y estrategias de afrontamiento en docentes de primaria y secundaria. Revista Colombiana de Ciencias Sociales, 3(2), 226-242.

Pizarro, J. A. (2010). La violencia escolar hacia el profesor. Autodidacta, Revista Online. Recuperado de http://www.anpebadajoz.es/ autodidacta/autodidacta_archivos/numero _5_archivos/1_j_a_p_cruz.pdf

Ratto, A., García Pérez, R. C., Silva, M. I., \& González, M. C. (2015). El síndrome de quemarse por el trabajo y factores psicosociales en docentes de primaria de la ciudad de Montevideo. Ciencias Psicológicas, 9(2), 273-281.

Sánchez, P. (2010). Cómo afrontar el estrés docente. Recuperado de http://pisaal.blogia.com/201 0/112601-investigacion-sobre-estres-docen te.-datos-de-la-investigacion.php

Tejero, A., Guimerá, E., Farré, J. M., \& Peri, J. M. (1986). Uso clínico del HAD (Hospital Anxiety and Depression Scale) en población psiquiátrica: un estudio de su sensibilidad, fiabilidad y validez. Revista del Departamento de Psiquiatría de la Facultad de Medicina de Barcelona, 13, 233-238.

Veas, C. (marzo/abril, 2013). Violencia en los colegios y agresiones a profesores. Revista de Pedagogía, 30-35.

Yuste, J. (2007). Bullying: acoso escolar. Recuperado de http://www.conflictoescolar .es/conflictividad-en-el-aula-violencia-con tra-el-profesora/

Zigmon, A. S., \& Snaith, R. P. (1983). The Hospital Anxiety and Depression Scale. Acta Psychiatrica Scandinavica, 67, 361-70.

\section{Notas}

* Artículo de investigación.

1 Centros escolares de origen privado pero que reciben subvenciones de la Administración Central 